\title{
Bariatric Arterial Embolization: Position Statement by the Pan Arab Interventional Radiology and the Saudi Interventional Radiology Societies
}

\section{Preface}

These guidelines are developed based on the Grading of Recommendations Assessment, Development, and Evaluation (GRADE) system. ${ }^{[1]}$ Grading strength of recommendations and quality of evidence is based on the UpToDate classification [Appendix A]. ${ }^{[2]}$ The scientific committees of the Pan Arab Interventional Radiology Society (PAIRS) and the Saudi Interventional Radiology Society (SIRS) nominated the guidelines development group (GDG) based on their expertise in the field of vascular interventional radiology and bariatric surgery from both private and academic institutions. Members are selected from different countries to further represent wide geographic distribution and institutional variations in practice within the Middle East and North Africa (MENA) region.

\section{Definitions and Abbreviations}

1. Obesity: The World Health Organization (WHO) defines obesity as a body mass index $(\mathrm{BMI}) \geq 30 \mathrm{~kg} / \mathrm{m}^{2}$
a. Obesity Class I: BMI of $30.0-34.9 \mathrm{~kg} / \mathrm{m}^{2}$
b. Obesity Class II: BMI $35.0-39.9 \mathrm{~kg} / \mathrm{m}^{2}$
c. Obesity Class III: BMI Above $40 \mathrm{~kg} / \mathrm{m}^{2}$.

2. Overweight: $\mathrm{BMI} \geq 25 \mathrm{~kg} / \mathrm{m}^{2}$

3. Ideal body weight (IBW): There are several methods for calculating IBW. Based on Lorentz formula, ${ }^{[3]}$ IBW is calculated as follows:
a. Males: IBW $=$ (height [cm]-100)-([height $(\mathrm{cm})-150]) / 4$
b. Females: IBW $=$ (height [cm]-100)-([height $(\mathrm{cm})-150]) / 2$.

4. Excess body weight: Current weight - ideal weight

\footnotetext{
This is an open access journal, and articles are distributed under the terms of the Creative Commons Attribution-NonCommercial-ShareAlike 4.0 License, which allows others to remix, tweak, and build upon the work non-commercially, as long as appropriate credit is given and the new creations are licensed under the identical terms.
}

5. Weight changes are expressed in the literature using several parameters including:
a. Percentage
total
weight
loss $=([$ Pretreatment total weight - post treatment total weight]/ Pretreatment total weight)
b. Percentage excess weight loss $($ EWL $)=([$ Pretreatment excess weight - Posttreatment excess weight]/Pretreatment excess weight) $\times 100$

c. Percentage BMI reduction: ([Pretreatment BMI - Posttreatment $\mathrm{BMI}] /$ pretreatment BMI) $\times 100$

d. Waist circumference change ${ }^{[4]}$

e. Weight-to-height ratio change ${ }^{[4]}$

f. Total body fat area $=$ Subcutaneous + visceral + intramuscular fat areas ${ }^{[3]}$

g. Total abdominal adipose tissue $=$ Subcutaneous + visceral fat $\operatorname{areas}^{[4]}$

h. Subcutaneous fat area: Determined on computerized tomography/ magnetic resonance imaging (CT/ MRI) using the outer boundary of the abdominal wall muscles and paraspinal muscles ${ }^{[3,4]}$

i. Visceral fat area: Determined on CT/MRI using the inner boundary of the abdominal wall muscles and paraspinal muscles ${ }^{[3,4]}$

j. Intramuscular fat: Determined on CT using the threshold attenuation values for fat (between - 190 and $30 \mathrm{HU}$ ) within the skeletal muscle compartments. ${ }^{[3]}$

6. Ghrelin: A neuropeptide predominantly produced in the gastric fundus, which

\section{Address for correspondence:}

Dr. Mohammad Arabi,

Department of Medical Imaging, Division of Vascular and Interventional Radiology, Ministry of National Guard-Health Affairs, Riyadh, Saudi Arabia.

E-mail:marabi2004@hotmail.com

How to cite this article: Arabi M, Abd El Tawab KA, Almoaiqel M, Chamsuddin A, Ashour M, Gunn AJ, et al. Bariatric arterial embolization: Position statement by the pan Arab interventional radiology and the Saudi interventional radiology societies. Arab J Intervent Radiol 2020;4:4-10.

\section{Mohammad \\ Arabi ${ }^{1,2,3}$, \\ Karim A. Abd El \\ Tawab4, \\ Mohammad \\ Almoaiqel ${ }^{1,2,3}$, \\ Abbas \\ Chamsuddin ${ }^{5}$, Majed Ashour ${ }^{6}$, Andrew J. Gunn? Ali Alzahrani ${ }^{2,3,8}$}

${ }^{1}$ Department of Medical Imaging, Division of Vascular and Interventional Radiology, Ministry of National Guard Health Affairs, ${ }^{2}$ King Abdullah International Medical Research Center, ${ }^{3}$ King Saud Bin Abdulaziz University for Health Sciences, Riyadh, ${ }^{6}$ Department of Radiology, Division of Vascular and Interventional Radiology, King Faisal Specialist Hospital and Research Center, Jeddah, Saudi Arabia, ${ }^{4}$ Department of Radiology, Interventional Radiology Unit, Ain Shams University Hospitals, Cairo, Egypt, ${ }^{5}$ Department of Radiology, Saint George Hospital University Medical Center, Beirut, Lebanon, ${ }^{7}$ Department of Radiology, Division of Vascular and Interventional Radiology, University of Alabama at Birmingham, Birmingham, Alabama, USA, ${ }^{8}$ Department of Surgery, Ministry of National Guard Health Affairs, Riyadh, Saudi Arabia

Received: 22-11-2019 Accepted: 22-11-2019 Published Online: 18-12-2019

\section{Access this article online}

Website: www.arabjir.com

DOI: 10.4103/AJIR.AJIR_31_19 Quick Response Code:

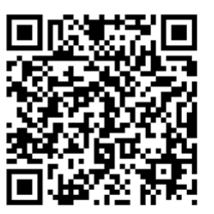


stimulates growth hormone secretion and food intake, and is considered a primary regulator of appetite ${ }^{[5]}$ Other hormones (cholecystokinin, leptin, peptide YY3-36, and glucagon-like peptide-1) involved in satiety control are discussed in depth in literature ${ }^{[5]}$

7. Bariatric arterial embolization (BAE): This technique entails transarterial embolization of the arterial supply to the gastric fundus using various embolic agents for bariatric indications. BAE term encompasses embolization of vessels other than the left gastric artery, such as the right gastroepiploic artery

8. Left gastric artery embolization (LGAE): This refers specifically to isolated left gastric artery transcatheter embolization

9. Gastric artery chemical embolization: Refers to LGAE using chemical liquid embolics such as morrhuate sodium $^{[6,7]}$

10. BEAT Obesity trial: Bariatric Embolization of Arteries for the Treatment of Obesity ${ }^{[8,9]}$

11. GET-LEAN trial: Gastric Artery Embolization Trial for the Lessening of Appetite Nonsurgically ${ }^{[10]}$

12. EMBARGO trial: Embolization of arterial gastric supply in obesity. ${ }^{[11]}$

\section{Methods}

A comprehensive literature review was conducted using the medical subheading (MESH) terms and Boolean operators searching "bariatric" AND "embolization," then "LGAE" AND "bariatric." After eliminating the irrelevant titles, a total of 34 records were retrieved including reviews, commentaries, preclinical, retrospective, prospective studies, and case series/reports. Literature search was updated on October 24, 2019 before the final release of the document. The articles were distributed to all members before drafting the guidelines and recommendations. Initial draft was circulated between the GDG members and underwent several rounds of discussion and review using online platforms. The document was then refereed for external expert review for further evaluation and comments (A. J. Gunn). The final document was reviewed and endorsed by the scientific committees and received final approval by the executive boards of both PAIRS and SIRS.

\section{Aim}

The prevalence of obesity is increasing in the MENA over the past several decades. ${ }^{[12]}$ For example, more than $30 \%$ of the adult population are considered obese in Kuwait, Jordan, Saudi Arabia, Qatar, Libya, Turkey, Lebanon, Egypt, United Arab Emirates, and Iraq. ${ }^{[12]}$ As such, dietary, surgical, and endoscopic bariatric interventions are on the rise in the Arab World and Gulf countries in attempt to contain this epidemic. ${ }^{[13]}$ Recently, bariatric arterial embolization (BAE) has been introduced as a minimally invasive procedure for appetite control and weight loss. However, the scarcity of evidence and lack of standardized protocols for this intervention necessitate careful evaluation.
The aim of this document is to provide a position statement on BAE by PAIRS and the SIRS and to propose a protocol for future research studies on BAE.

\section{Background}

The endocrine function of the gastric fundus in regulating satiety and body weight is predominantly controlled by Ghrelin, which stimulates growth hormone secretion and food intake. Several other hormones produced by the gastrointestinal (GI) tract are involved in metabolic homeostasis. ${ }^{[5]}$ Early preclinical animal studies have identified a link between suppression of plasma concentrations of Ghrelin and devascularization of the gastric fundus by occlusion of the left gastric artery by means of chemical embolization, ${ }^{[6,7]}$ particulate embolics, ${ }^{[11,14-19]}$ surgical clipping, ${ }^{[20]}$ or radioembolization with Y90. ${ }^{[21]}$ This results in decreased appetite and weight Modulation as a result of reducing Ghrelin-expressing cells in the gastric fundus. ${ }^{[6,7,11,14-18,20-22]}$

Early weight loss was reported in patients with GI bleeding who underwent LGAE compared to those who had embolization of different mesenteric vessels. ${ }^{[23-25]}$ Body composition analysis following LGAE for gastric bleeding indicated significant decrease in subcutaneous and total body fat as well as skeletal muscle index leading to significant decrease in body weight and BMI. ${ }^{[3]}$

\section{Effects of BAE on weight}

The currently reported retrospective studies and prospective trials included obese patients with mean BMI range between 38 and $52 \mathrm{~kg} / \mathrm{m}^{2}$ and reported short-term clinical outcomes.

In a retrospective study on patients who underwent LGAE for GI bleeding indications, the LGAE group lost an average of $7.3 \%$ of initial body weight at 3 months after embolization, which was significantly greater than the $2 \%$ weight loss observed in the control group of other mesenteric embolization. ${ }^{[4]} \mathrm{Kim}$ et al. reported a median of $16.3 \mathrm{~kg}$ weight loss over a median time of 12 months (range, 2-72 month) following LGAE in cancer naïve patients with upper GI bleeding. ${ }^{[23]}$ Similarly, Kevin Anton et al. reported significant early (1 and 4 month) weight loss after LGAE for bleeding indications; however, weight change was not significant at 8 and 12 month compared to the control group of other mesenteric arterial embolization. ${ }^{[25]}$

The GET-LEAN trial reported average EWL at 6 months of $17.2 \%{ }^{[10]}$ Bai et al. demonstrated significant average weight loss of $10.4 \mathrm{~kg}$ at 6 month following BAE. ${ }^{[4]}$ The BEAT trial, which enrolled 20 participants with mean BMI of $45 \mathrm{~kg} / \mathrm{m}^{2}$, showed that BAE resulted in significant weight loss of $11.5 \%$ at 6 months, which was maintained until 12 months of follow-up. Kipshidze et al. reported that all 5 patients maintained significant weight loss of 
$17 \%$ at $20-24$ months post BAE. ${ }^{[26]}$ Zaitoun et al. reported significant BMI reduction of $8.8 \%$ at 6 months following the procedure. ${ }^{[27]}$ Elens et al. evaluated BAE in overweight patients with mean baseline BMI of $28.9 \mathrm{~kg} / \mathrm{m}^{2}$. The mean early weight loss in 9 patients at 6 months was 8 $\mathrm{kg}$, which corresponded to $10 \%$ of their baseline weight. One-year follow-up was available in 3 patients who maintained mean weight loss of $9.66 \mathrm{~kg} .{ }^{[28]}$ In a recent pooled analysis ${ }^{[29]}$ of 47 patients in 6 studies, ${ }^{[4,8,10,26,28,30]}$ BAE resulted in statistically significant mean absolute weight loss of $8.85 \mathrm{~kg}(7.6-22 \mathrm{~kg})$ correlating to mean percentage weight loss of $8.1 \%(4.7 \%-17 \%)$ at 12 -month follow-up. Male sex was associated with greater weight loss compared to females. ${ }^{[29]}$

\section{Effects of BAE on glycemic control and lipid profile}

The HgbA1c-lowering effect of BAE is demonstrated in few reports. ${ }^{[8,10,27]}$ Zaitoun et al. evaluated the effect of BAE on 10 obese prediabetic patients with baseline BMI of $37.4 \mathrm{~kg} / \mathrm{m}^{2}$ and mean HgbA1c of 6 . There was statistically significant reduction in mean BMI and $\mathrm{HbAlc}$ of $8.8 \%$ and $21.4 \%$, respectively. In the BEAT study, the mean total cholesterol and low-density lipoprotein levels were lower at 12 months compared to their respective means at baseline. Conversely, the high-density lipoprotein was significantly higher at 12-month follow-up. Mean triglycerides initially decreased at 1 month, then increased back to baseline levels at 3, 6, and 12 months. Hemoglobin A1c at 12 months was significantly lower than baseline but did not correlate with weight change. ${ }^{[8]}$ Although there is a favorable signal to improved lipid profile and glycemic control during the $1^{\text {st }}$ month after BAE, these changes require careful evaluation in future studies.

\section{Candidates for bariatric artery embolization}

- Patients with severe or morbid obesity (WHO grade $3, \mathrm{BMI}>40 \mathrm{~kg} / \mathrm{m}^{2}$ ), or with complications of obesity should be offered surgical interventions first. BAE may be offered as alternative option for nonsurgical candidates or those who refuse surgical procedure (Grade 2C)

- We suggest limiting BAE to individuals with WHO grade $\geq 2$ obesity to achieve clinically meaningful weight loss (Grade 2C)

- There is limited evidence studying the metabolic effects of BAE, such as improvements in glycemic control. Therefore, BAE should not replace proven medical or surgical therapies for patients with diabetes or prediabetes

- Nutritional consultation is vital for all patients before and following BAE. Patients with a known history of eating disorders should not undergo BAE

- Patients with active or a history of peptic ulcer disease should not undergo BAE

- Patients with prior surgery to the stomach or small intestine should not undergo BAE.

\section{Effects on quality of life}

Weight changes after BAE are correlated with decreased hunger scores and improved QoL as demonstrated by the GET-LEAN and BEAT trials. ${ }^{[8,10]}$ The psychological impact of weight changes, waist circumference decrease, and improvement in mental and physical scores may help in maintaining appetite control and lifestyle modifications. Nonetheless, QoL and other mental scores need to be carefully evaluated in future studies and compared to other methods of weight reduction.

\section{Effects of $\mathrm{BAE}$ on gastric mucosa}

One of the main concerns in BAE is the sequelae of gastric ischemia and the histologic changes induced by BAE. Initial animal studies demonstrated reduction in Ghrelin-expressing cells in the gastric fundus with preservation of the overall architecture and parietal cells, ${ }^{[7,16]}$ with a trend toward increased fibrosis in the gastric fundus ${ }^{[16]}$ Although mucosal gastric ulcers are reported post gastric artery embolization in both animal ${ }^{[16,19,21]}$ and human studies, ${ }^{[4,8,9,23,28,31]}$ majority of ulcers healed spontaneously with no major complications. Only single case of gastric perforation and splenic infarction was reported following BAE with 500-700 polyvinyl alcohol (PVA) particles. ${ }^{[28]}$ The use of smaller particles $(100-300 \mu)$ appears to induce greater weight loss, but with more gastric ulcerations. ${ }^{[19]}$ The administration of gastroprotective agents and embolization of fewer arteries to the gastric fundus did not prevent gastric ulceration in porcine models. ${ }^{[15]}$

\section{Gastrointestinal protection}

- We recommend the use of gastroprotective agents (oral omeprazole $40 \mathrm{mg}$ twice daily and sucralfate $1 \mathrm{~g}$ four times daily) 2 weeks before BAE and 6 weeks after (Grade 2C)

- It is advised to perform upper endoscopy prior to BAE and within 4 weeks after procedure to further document the impact of gastric embolization on gastric mucosa (Grade 2C)

- We suggest a single dose of pre procedure prophylactic antibiotics.

Effects of BAE on fundal vascularity and future bariatric surgery

Diana et al. performed surgical clipping of the gastric artery in porcine models and demonstrated increased vascular anastomotic network in the fundus in 2 of pigs with no noticeable change in the remaining 3 animals. ${ }^{[20]}$ The EMBARGO trial, which investigated the possibility of sleeve gastrectomy after LGAE, suggested that embolization may enhance the vascular supply to the gastroesophageal junction in preparation for sleeve procedure. ${ }^{[11]}$

Although some authors caution that BAE may preclude future bariatric surgery due to potential compromise of 
fundal vascularity, ${ }^{[32,33]}$ the evidence to support or refute this concern remains not investigated. In human subjects, there is a single reported case of bariatric surgery 2 years after bariatric embolization. ${ }^{[28]}$ None of the existing human studies evaluated fundal vascularity by catheter or CT angiography following BAE.

\section{$B A E$ and future bariatric surgery}

- Due to concerns about the safety of bariatric surgery following BAE, the GDG recommends careful multidisciplinary patient selection and counseling of patients who may require future bariatric surgery

- Patients who will undergo bariatric surgery after BAE may require evaluation of the gastric arterial supply by catheter or CT angiography to determine the patency of fundal supply.

\section{Proposed BAE technique}

Access

Avoiding puncture site complications is of particular importance in morbidly obese patients. Femoral artery access in morbidly obese patients is shown to be associated with greater risk for bleeding and access site complications after coronary interventions. ${ }^{[34-36]}$ Therefore, transradial approach has become the standard access for coronary interventions particularly in this subset of patients. Adopting transradial access (TRA) for visceral interventions is gaining increasing popularity ${ }^{[34-36]}$ as it is associated with improved patient experience and shorter recovery times; however, it can be more technically challenging and may require longer procedure/fluoroscopy times. ${ }^{[37,38]}$ Pirlet et al. performed a pilot study to evaluate the feasibility, safety, and efficacy of LGAE using TRA. ${ }^{[30]}$ The mean fluoroscopy time in their study was $10 \mathrm{~min}$, which appears shorter than what is reported in the GET-LEAN study $(26 \mathrm{~min})^{[10]}$ and the BEAT study (31 min). ${ }^{[8]}$ No puncture site complications were reported in neither femoral nor radial access BAE interventions.

- The choice of arterial access should be at the discretion of the operator's expertise. However, it is advised to use radial access for BAE interventions whenever possible to improve patient satisfaction and minimize vascular complications associated with morbid obesity. Availability of TRA expertise and equipment is paramount to ensure shorter fluoroscopy times and lower radiation exposures (Grade 1A).

\section{Choice of embolic agent}

Various methods have been reported for left gastric artery occlusion for bariatric or bleeding intentions including surgical clipping, chemical sclerosants, gelfoam, coils, Y90 radioembolization, and most commonly particulate embolics. Bariatric embolization should achieve sufficient devascularization of the gastric fundus and Ghrelin-producing cells without resulting in mucosal ischemia and ulcerations. Larger particles may aggregate in the proximal vessel and may result in less devascularization of the target cells due to collateral filling from other arterial anastomosis. On the other hand, smaller particles may lead to more gastric ulcers but associated with greater weight loss as demonstrated in animal study by Fu et al. ${ }^{[19]}$

In the clinical studies on BAE, particulate size was $300-500 \mu^{[8-10,26,27,30,31]}$ or 500-700 $\mu .^{[4,28]}$ Only single case of gastric perforation is reported with the use of 500-700 $\mu$ PVA particles, without details on the embolization procedure.

- Comparative safety and efficacy data on the choice of embolic agent and particulate size remain lacking and require further research

- We suggest to use particulate size of $100-300$ or $300-500 \mu$ until further evidence is available (Grade 2C)

- Proximal embolization with coils or vascular plugs should be avoided as it may preclude future embolization and may not be sufficient for fundal devascularization due to collateral anastomosis (Grade 2C)

Number of embolized vessels

LGAE was performed in all previously reported studies. ${ }^{[4,8-10,26-28,30,31]}$ The gastroepiploic artery was embolized in 3 patients in the BEAT study. ${ }^{[9]}$

- We recommend careful evaluation and mapping of the fundal arterial supply before bariatric embolization (Grade 1A). It is advised to perform pre procedure CT angiography to assess the vascular anatomy and determine the arterial access

- Identification of left gastric artery variants is essential to achieve proper embolization of the fundus

- Gastroepiploic artery embolization may be considered if fundal blush is deemed incomplete on the left gastric artery angiography

- It is advised to perform selective cannulation of the fundal branches to minimize the risk of mucosal ulceration and non-target embolization such as to the pancreas or spleen.

Postprocedure care and follow-up

- We recommend the use of gastroprotective agents 2 weeks before BAE and 6 weeks after

- We recommend close monitoring of weight changes to determine the effectiveness of BAE. Long-term outcomes are needed to determine the durability of BAE

- We recommend strict adherence to dietary regimens to further augment the benefits of BAE

- Ghrelin monitoring is advised, particularly in the context of research protocols

- Periodic HgbAlc and lipid profile monitoring is recommended in prediabetic and diabetic patients 
- It is advised to perform upper endoscopy before BAE and within 4 weeks after procedure to further document the impact of gastric embolization on gastric mucosa (Grade 2C).

\section{Conclusion}

There is scarce evidence that indicates the safety and effectiveness of BAE in achieving early weight reduction and possibly glycemic control. The intermediate and long-term effects of BAE in maintaining Ghrelin levels and weight loss are unknown yet. The ideal BMI of candidates remains unclear, and additional studies are still required. The GDG concludes that BAE should only be conducted in the context of clinical trials and proposes the aforementioned protocol recommendations based on the existing preclinical and clinical evidence as well as authors' expertise in the field.

\section{Disclaimer}

These guidelines serve as an educational resource to help practicing physicians in their approach to candidates for bariatric arterial embolization and to promote highquality clinical practice and research. These guidelines are drafted based on currently available evidence, and recommendations may change over time as new evidence emerges. This document is not intended to be a legal standard of care and its use should be at the discretion of the practicing physicians. PAIRS and SIRS are not responsible for any clinical actions taken based on these guidelines.

\section{Financial support and sponsorship}

Nil.

\section{Conflicts of interest}

There are no conflicts of interest.

\section{References}

1. Guyatt GH, Oxman AD, Vist GE, Kunz R, Falck-Ytter Y, Alonso-Coello P, et al. GRADE: An emerging consensus on rating quality of evidence and strength of recommendations. BMJ 2008;336:924-6.

2. UpToDate. Grading Guide. UpToDate. Grading Guide. Available from: https://www.uptodate.com/home/gradingguide\#GradingRecommendations. [Last accessed on 2019 Nov 22].

3. Takahashi EA, Takahashi N, Reisenauer CJ, Moynagh MR, Misra S. Body composition changes after left gastric artery embolization in overweight and obese individuals. Abdom Radiol (NY) 2019;44:2627-31.

4. Bai ZB, Qin YL, Deng G, Zhao GF, Zhong BY, Teng GJ. Bariatric embolization of the left gastric arteries for the treatment of obesity: 9-month data in 5 patients. Obes Surg 2018;28:907-15.

5. Weiss CR, Gunn AJ, Kim CY, Paxton BE, Kraitchman DL, Arepally A. Bariatric embolization of the gastric arteries for the treatment of obesity. J Vasc Interv Radiol 2015;26:613-24.

6. Arepally A, Barnett BP, Patel TH, Howland V, Boston RC,
Kraitchman DL, et al. Catheter-directed gastric artery chemical embolization suppresses systemic ghrelin levels in porcine model. Radiology 2008;249:127-33.

7. Arepally A, Barnett BP, Montgomery E, Patel TH. Catheter-directed gastric artery chemical embolization for modulation of systemic ghrelin levels in a porcine model: Initial experience. Radiology 2007;244:138-43.

8. Weiss CR, Abiola GO, Fischman AM, Cheskin LJ, Vairavamurthy J, Holly BP, et al. Bariatric embolization of arteries for the treatment of obesity (BEAT Obesity) trial: Results at 1 year. Radiology 2019;291:792-800.

9. Weiss CR, Akinwande O, Paudel K, Cheskin LJ, Holly B, Hong K, et al. Clinical Safety of Bariatric Arterial Embolization: Preliminary Results of the BEAT Obesity Trial. Radiology 2017;283:598-608.

10. Syed MI, Morar K, Shaikh A, Craig P, Khan O, Patel S, et al. Gastric artery embolization trial for the lessening of appetite nonsurgically (GET LEAN): Six-month preliminary data. J Vasc Interv Radiol 2016;27:1502-8.

11. Diana M, Pop R, Beaujeux R, Dallemagne B, Halvax P, Schlagowski I, et al. Embolization of arterial gastric supply in obesity (EMBARGO): An endovascular approach in the management of morbid obesity. Proof of the concept in the porcine model. Obes Surg 2015;25:550-8.

12. Word Health Organization. Prevalence of Obesity among Adults, Ages 18+, 1975-2016 (Age Standardized Estimate): Both Sexes, 2016. Word Health Organization; 2016.

13. AlMarri F, Al Sabah S, Al Haddad E, Vaz JD. A call for more research from the Arabian Gulf. Obes Surg 2017;27:2034-43.

14. Kim JM, Kim MD, Han K, Muqmiroh L, Kim SU, Kim GM, et al. Bariatric arterial embolization with non-spherical polyvinyl alcohol particles for ghrelin suppression in a swine model. Cardiovasc Intervent Radiol 2017;40:744-9.

15. Paxton BE, Arepally A, Alley CL, Kim CY. Bariatric embolization: Pilot study on the impact of gastroprotective agents and arterial distribution on ulceration risk and efficacy in a porcine model. J Vasc Interv Radiol 2016;27:1923-8.

16. Paxton BE, Alley CL, Crow JH, Burchette J, Weiss CR, Kraitchman DL, et al. Histopathologic and immunohistochemical sequelae of bariatric embolization in a porcine model. J Vasc Interv Radiol 2014;25:455-61.

17. Paxton BE, Kim CY, Alley CL, Crow JH, Balmadrid B, Keith CG, et al. Bariatric embolization for suppression of the hunger hormone ghrelin in a porcine model. Radiology 2013;266:471-9.

18. Bawudun D, Xing Y, Liu WY, Huang YJ, Ren WX, Ma M, et al. Ghrelin suppression and fat loss after left gastric artery embolization in canine model. Cardiovasc Intervent Radiol 2012;35:1460-6.

19. Fu Y, Weiss CR, Paudel K, Shin EJ, Kedziorek D, Arepally A, et al. Bariatric arterial embolization: Effect of microsphere size on the suppression of fundal ghrelin expression and weight change in a swine model. Radiology 2018;289:83-9.

20. Diana M, Halvax P, Pop R, Schlagowski I, Bour G, Liu YY, et al. Gastric supply manipulation to modulate ghrelin production and enhance vascularization to the cardia: Proof of the concept in a porcine model. Surg Innov 2015;22:5-14.

21. Pasciak AS, Bourgeois AC, Paxton BE, Nodit L, Coan PN, Kraitchman D, et al. Bariatric radioembolization: A pilot study on technical feasibility and safety in a porcine model. J Vasc Interv Radiol 2016;27:1509-17.

22. Anton K, Rahman T, Bhanushali A, Patel AA. Bariatric left gastric artery embolization for the treatment of obesity: A review 
of gut hormone involvement in energy homeostasis. AJR Am J Roentgenol 2016;206:202-10.

23. Kim DJ, Raman HS, Salter A, Ramaswamy R, Gunn AJ, Weiss CR, et al. Analysis of weight changes after left gastric artery embolization in a cancer-naive population. Diagn Interv Radiol 2018;24:94-7.

24. Gunn AJ, Oklu R. A preliminary observation of weight loss following left gastric artery embolization in humans. J Obes 2014;2014:185349.

25. Kevin Anton TR, Bhanushali AB, Nadal LL, Pierce G, Patel AA. Weight loss following left gastric artery embolization in a human population without malignancy: A retrospective review. J Obesity Weight Loss Therapy 2015;5:285.

26. Kipshidze N, Archvadze A, Bertog S, Leon MB, Sievert H. Endovascular bariatrics: First in humans study of gastric artery embolization for weight loss. JACC Cardiovasc Interv 2015;8:1641-4.

27. Zaitoun MM, Basha MA, Hassan F, Elsayed SB, Farag AA, Amer M, et al. Left gastric artery embolization in obese, prediabetic patients: A pilot study. J Vasc Interv Radiol 2019;30:790-6.

28. Elens S, Roger T, Elens M, Rommens J, Sarafidis A, Capelluto E, et al. Gastric embolization as treatment for overweight patients; efficacy and safety. Cardiovasc Intervent Radiol 2019;42:513-9.

29. Hafezi-Nejad N, Bailey CR, Gunn AJ, Weiss CR. Weight loss after left gastric artery embolization: A systematic review and meta-analysis. J Vasc Interv Radiol 2019;30:1593-603.

30. Pirlet C, Ruzsa Z, Costerousse O, Nemes B, Merkely B, Poirier P, et al. Transradial left gastric artery embolization to treat severe obesity: A pilot study. Catheter Cardiovasc Interv 2019;93:365-70.

31. Salsamendi J, Pereira K, Kang K, Fan J. Minimally invasive percutaneous endovascular therapies in the management of complications of non-alcoholic fatty liver disease (NAFLD): A case report. J Radiol Case Rep 2015;9:36-43.

32. Fink JM, Martini V, Seifert G, Marjanovic G. Left gastric artery embolization for weight loss-a dead-end procedure. Obes Surg 2018;28:3623-4.

33. Kordzadeh A, Lorenzi B, Hanif MA, Charalabopoulos A. Left gastric artery embolisation for the treatment of obesity: A systematic review. Obes Surg 2018;28:1797-802.

34. Hibbert B, Simard T, Wilson KR, Hawken S, Wells GA, Ramirez FD, et al. Transradial versus transfemoral artery approach for coronary angiography and percutaneous coronary intervention in the extremely obese. JACC Cardiovasc Interv 2012;5:819-26.

35. Byrne J, Spence MS, Fretz E, Mildenberger R, Chase A, Berry $\mathrm{B}$, et al. Body mass index, periprocedural bleeding, and outcome following percutaneous coronary intervention (from the British Columbia Cardiac Registry). Am J Cardiol 2009;103:507-11.

36. Cox N, Resnic FS, Popma JJ, Simon DI, Eisenhauer AC, Rogers C. Comparison of the risk of vascular complications associated with femoral and radial access coronary catheterization procedures in obese versus nonobese patients. Am J Cardiol 2004;94:1174-7.

37. Hung ML, Lee EW, McWilliams JP, Padia SA, Ding P, Kee ST. A reality check in transradial access: A single-centre comparison of transradial and transfemoral access for abdominal and peripheral intervention. Eur Radiol 2019;29:68-74.

38. Yamada R, Bracewell S, Bassaco B, Camacho J, Anderson MB, Conrad A, et al. Transradial versus transfemoral arterial access in liver cancer embolization: Randomized trial to assess patient satisfaction. J Vasc Interv Radiol 2018;29:38-43. 


\begin{tabular}{ll}
\hline & Appendix A: UpToDate grading recommendations \\
\hline Grade of & Clarity of risk/benefit $\quad$ Quality of supporting evidence
\end{tabular}

recommendation

1A. Strong Benefits clearly outweigh Consistent evidence from well-performed

recommendation, risk and burdens, or vice

high-quality versa

evidence

versa

randomized, controlled trials or overwhelming evidence of some other form. Further research is unlikely to change our confidence in the estimate of benefit and risk

\section{B. Strong} recommendation, Benefits clearly outweigh moderate-quality risk and burdens, or vice evidence

\section{C. Strong recommendation, low-quality evidence

$$
\begin{aligned}
& \text { Benefits appear to } \\
& \text { outweigh risk and } \\
& \text { burdens, or vice versa }
\end{aligned}
$$

\section{A. Weak} recommendation, high-quality evidence

\section{B. Weak} recommendation, moderate-quality evidence

\section{C. Weak} recommendation, low-quality evidence
Benefits closely balanced with risks and burdens

Benefits closely balanced with risks and burdens, some uncertainly in the estimates of benefits, risks, and burdens

Uncertainty in the estimates of benefits, risks, and burdens; benefits may be closely balanced with risks and burdens
Evidence from randomized, controlled trials with important limitations (inconsistent results, methodological flaws, indirect or imprecise), or very strong evidence of some other research design. Further research (if performed) is likely to have an impact on our confidence in the estimate of benefit and risk and may change the estimate

Evidence from observational studies, unsystematic clinical experience, or from randomized, controlled trials with serious flaws. Any estimate of effect is uncertain

Consistent evidence from well-performed randomized, controlled trials or overwhelming evidence of some other form. Further research is unlikely to change our confidence in the estimate of benefit and risk

Evidence from randomized, controlled trials with important limitations (inconsistent results, methodological flaws, indirect or imprecise), or very strong evidence of some other research design. Further research (if performed) is likely to have an impact on our confidence in the estimate of benefit and risk and may change the estimate

Evidence from observational studies, unsystematic clinical experience, or from randomized, controlled trials with serious flaws. Any estimate of effect is uncertain

\section{Implications}

Strong recommendations: can apply to most patients in most circumstances without reservation. Clinicians should follow a strong recommendation unless a clear and compelling rationale for an alternative approach is present

Strong recommendation: applies to most patients. Clinicians should follow a strong recommendation unless a clear and compelling rationale for an alternative approach is present

Strong recommendation: applies to most patients. Some of the evidence base supporting the recommendation is, however, of low quality

Weak recommendation: best action may differ depending on circumstances or patients or societal values

Weak recommendation: alternative approaches likely to be better for some patients under some circumstances

Very weak recommendation: other alternatives may be equally reasonable 